\title{
Feedback by massive stars and the emergence of superbubbles
}

\author{
II. X-ray properties $\star$ \\ Martin Krause ${ }^{1,2}$, Roland Diehl ${ }^{1,2}$, Hans Böhringer ${ }^{1,2}$, Michael Freyberg ${ }^{1}$, and Daniel Lubos ${ }^{1}$ \\ ${ }^{1}$ Max-Planck-Institut für extraterrestrische Physik, Giessenbachstr. 1, 85741 Garching, Germany \\ e-mail: krause@mpe.mpg.de \\ 2 Excellence Cluster Universe, Technische Universität München, Boltzmannstrasse 2, 85748 Garching, Germany
}

Received 24 March 2014 / Accepted 29 April 2014

\begin{abstract}
Context. In a previous paper we investigated the energy transfer of massive stars to the interstellar medium (ISM) as a function of time and the geometrical configuration of three massive stars via 3D-mesh-refining hydrodynamics simulations, following the complete evolution of the massive stars and their supernovae with the exception of non-thermal processes.

Aims. To compare our results against observations we derive thermal X-ray properties of the ISM from our simulations and compare them to observations of superbubbles in general, to the well-studied nearby Orion-Eridanus superbubble and to the diffuse soft X-ray emission of nearby galaxies.

Methods. We analysed our ISM simulation results with the help of spectra for plasma temperatures between 0.1 and $10 \mathrm{keV}$ and computed the spectral evolution and the spatio-temporal distribution of the hot gas.

Results. Despite significant input of high-temperature gas from supernovae and fast stellar winds, the resulting thermal X-ray spectra are generally very soft, with most of the emission well below $1 \mathrm{keV}$. We show that this is due to mixing triggered by resolved hydrodynamic instabilities. Supernovae enhance the X-ray luminosity of a superbubble by $1-2$ orders of magnitude for a time span of about $0.1 \mathrm{Myr}$; which is longer if a supernova occurs in a larger superbubble and shorter in higher energy bands. Peak superbubble luminosities of the order of $10^{36} \mathrm{erg} \mathrm{s}^{-1}$ are reproduced well. The strong decay of the X-ray luminosity is due to bubble expansion, hydrodynamic instabilities related to the acceleration of the superbubble's shell thanks to the sudden energy input, and subsequent mixing. We also find global oscillations of our simulated superbubbles, which produce spatial variations of the X-ray spectrum, similar to what we see in the Orion-Eridanus cavity. We calculated the fraction of energy emitted in X-rays and find that with a value of a few times $10^{-4}$, it is about a factor of ten below the measurements for nearby galaxies.

Conclusions. Our models explain the observed soft spectra and peak X-ray luminosities of individual superbubbles. Each supernova event inside a superbubble produces a fairly similar heating-entrainment-cooling sequence, and the energy content of superbubbles is always determined by a specific fraction of the energy released by one supernova. For a given superbubble, soft X-rays trace the internal energy content well with moderate scatter. Some mechanism seems to delay the energy loss in real superbubbles compared to our simulations. Alternatively, some mechanism other than thermal emission of superbubbles may contribute to the soft X-ray luminosity of star-forming galaxies.
\end{abstract}

Key words. ISM: bubbles - ISM: structure - galaxies: ISM - hydrodynamics - instabilities - X-rays: ISM

\section{Introduction}

Stellar feedback is an essential ingredient in galaxy evolution models (e.g. Sommer-Larsen et al. 2003; Scannapieco et al. 2008, 2012; Elmegreen \& Burkert 2010; Piontek \& Steinmetz 2011; Henriques et al. 2013; Romeo Velonà et al. 2013): it regulates the star-formation rate (SFR), mass concentration and angular momentum distribution, the scale height of the interstellar medium (ISM; e.g. de Avillez \& Breitschwerdt 2005; Dobbs et al. 2011), and the chemical evolution of stars and gas (Böhringer \& Werner 2010; Putman et al. 2012). Predictions are, however, not based on feedback calculations from first principles, and different prescriptions for stellar feedback lead to the following major deviations in the properties of simulated galaxies (Elmegreen \& Burkert 2010; Scannapieco et al. 2012; Agertz et al. 2013): the resulting total stellar mass is uncertain by a

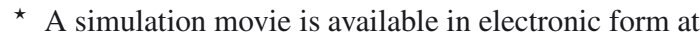
http: //www . aanda.org factor of a few; stars tend to be too concentrated for all prescriptions, which leads to unrealistically declining rotation curves; even the morphological type of a galaxy, i.e. whether or not it has a stellar disc, seems to depend on the feedback implementation. It is clear that stellar feedback is important for the evolution of all but perhaps the most massive galaxies, where supermassive black holes may dominate (e.g. Krause 2005; Croton et al. 2006; Nesvadba et al. 2008; Krause \& Gaibler 2010; Gaspari et al. 2012; Silk \& Mamon 2012).

Massive stars are the main agents of stellar feedback, and they form mainly in groups (Zinnecker \& Yorke 2007). Their energy output produces bubbles, shells and bipolar structures, which are observed in great detail in nearby star-forming complexes (e.g. Nielsen et al. 2009; Motte et al. 2010; Preibisch et al. 2012; Minier et al. 2013; Russeil et al. 2013). The compression of surrounding gas may trigger further star formation (Ohlendorf et al. 2012; Roccatagliata et al. 2013). Star-forming regions such as the Carina, Cygnus or Orion star-forming complexes form 
a total of around 100 massive stars $\left(>8 M_{\odot}\right.$ ) each (Knödlseder et al. 2002; Voss et al. 2010, 2012). Population synthesis (Voss et al. 2009) gauged by observations of the stellar content predicts the output of energy, which may be compared to the energy needed to create the observed bubbles. For example, in the case of Orion, the massive stars have output about $2 \times 10^{52} \mathrm{erg}$ of kinetic energy into the surrounding gas, which agrees with the amount of energy needed to create the Orion-Eridanus superbubble (Voss et al. 2010). Such superbubbles are prominent structures in the ISM of the Milky Way and nearby galaxies with features e.g. in molecular gas (Dawson et al. 2013), HI (socalled HI-holes, Boomsma et al. 2008; Bagetakos et al. 2011; Ehlerová \& Palouš 2013), H $\alpha$ (Rossa et al. 2004; Voigtländer et al. 2013), Gamma-ray lines (radioactive trace elements ejected from massive stars, Knödlseder et al. 2002; Diehl et al. 2006; Diehl 2013; Kretschmer et al. 2013), and Gamma-ray continuum due to cosmic rays (Ackermann et al. 2011).

Uncertainties are substantial in the study of feedback effects: for example, recent Herschel observations suggest that the number of massive stars may have been underestimated by up to a factor of two in the Carina nebula complex (Roccatagliata et al. 2013), uncertainties from stellar evolution calculations and wind prescriptions lead to an uncertainty of the energy output of tens of per cent (Voss et al. 2009, 2012). Uncertainties also exist in the coupling of the energy output of the massive stars to the ambient ISM: hydrodynamic simulations show that the major fraction of the injected energy is lost to radiation (e.g. Tenorio-Tagle et al. 1990; Thornton et al. 1998; Freyer et al. 2006; Creasey et al. 2013) and that the clustering may affect the energy retained in the gas by a factor of a few (Krause et al. 2013, hereafter Paper I).

In order to improve the accuracy of feedback modelling, it is therefore important to exploit all the available observational constraints. One such constraint is the diffuse soft X-ray emission, on which we focus here. In general, diffuse X-ray emission from star-forming regions may be associated with unresolved stars, cosmic ray acceleration and hot gas. Because superbubbles are much larger than the associated energy-liberating star-forming region, unresolved stars are only important where the starforming complex is observed directly (e.g. Muno et al. 2006). Cosmic rays are evidently accelerated in superbubbles. This follows directly from the Fermi detection of diffuse cosmic ray emission in the Cygnus superbubble (Ackermann et al. 2011), and is suggested by the high-energy detections of star-forming galaxies (e.g. Ackermann et al. 2012) and the infrared/radio correlation (e.g. Garn \& Alexander 2009; Schleicher \& Beck 2013). Cosmic rays are expected to take a share of a few, up to ten per cent of the energy injected by massive stars via diffusive shock acceleration (Schure et al. 2012; Vink 2012; Bell 2013; Rieger et al. 2013, for recent reviews). This process may work for shocks from winds and supernovae in various environments (e.g. Ellison et al. 2012), including superbubbles (Parizot et al. 2004; Butt \& Bykov 2008; Bykov et al. 2013). Non-thermal $\mathrm{X}$-ray spectra due to cosmic rays are detected in the diffuse emission associated with some massive star clusters, e.g. 30 Doradus (Bamba et al. 2004) and Westerlund 1 (Muno et al. 2006). However, many superbubbles do not show evidence for nonthermal X-ray emission (e.g. Yamaguchi et al. 2010). Where observed, the non-thermal emission is more important above $2 \mathrm{keV}$, and softer, thermal components may often be extracted from the X-ray spectra (e.g. Bamba et al. 2004; Muno et al. 2006). Hot gas emits diffuse soft X-ray emission in individual superbubbles (e.g. Sasaki et al. 2011; Kavanagh et al. 2012) and entire starforming galaxies (Strickland et al. 2004a). Here, we restrict our attention to the diffuse, soft X-ray emission due to hot gas, noting that cosmic rays are not explicitly accounted for in our analysis.

Even wind bubbles of isolated massive stars should be expected to produce hot gas due to the high wind velocities (>1000 km s${ }^{-1}$, e.g. Puls et al. 1996; Vink \& Gräfener 2012), especially in the high-power Wolf-Rayet phase (Gräfener et al. 2011), and thus to be X-ray bright. Indeed two such examples are known (Zhekov \& Park 2011; Toalá et al. 2012). The interaction of individual bubbles leads to the formation of superbubbles (Oey et al. 2001; Chu 2008; Oey 2009, for reviews): they reach sizes of several $100 \mathrm{pc}$. When they are X-ray-bright this is suspected to be related to recent supernova activity. Superbubbles usually have a thermal X-ray spectrum (e.g. Sasaki et al. 2011; Jaskot et al. 2011, compare above for non-thermal contributions) with typical temperatures around $0.1 \mathrm{keV}$ and luminosities of the order of $10^{35}-10^{36} \mathrm{erg} \mathrm{s}^{-1}$. They are often surprisingly bright compared to expectations from models (Oey \& GarcíaSegura 2004), which is usually explained by entrainment of mass due to interaction with the shell walls (Jaskot et al. 2011). Hydrodynamic instabilities play a key role in simulations of superbubbles (e.g. Breitschwerdt \& de Avillez 2006), but have so far not been quantitatively assessed regarding their effect on the general X-ray properties of superbubbles.

In Paper I, we simulated superbubbles emerging from three massive stars in a constant density environment. We plan to compare the results in detail to superbubble observations in various wavelength regimes. Here, we focus on X-rays: a key observational feature, which we reproduce well, is the large X-ray variability. A recent supernova may boost the X-ray luminosity by a factor of up to a hundred for a timescale of order $10^{5} \mathrm{yr}$. Regarding morphology, we compare to previously unpublished data from the nearby Orion-Eridanus superbubble. We interpret the observed systematic spectral variations with position on the sky as global oscillations of the hot gas inside the bubble. The temperature-luminosity diagram is compared to data from superbubbles in the Large Magellanic Cloud (LMC). While the general ranges of luminosities and temperatures show reasonable overlap, there are also some discrepancies. We then compare the total thermal X-ray emission integrated over the lifetime of a simulated superbubble as a fraction of the input energy to the corresponding number derived from the soft X-ray luminosity and the SFR for nearby galaxies. The simulations underpredict the data by a factor of ten. We discuss possible reasons for this in Sect. 5.

\section{Simulations and their analysis}

Our analysis is based on 3D adaptive-mesh-refining hydrodynamics simulations with the NIRVANA-code described in detail in Paper I. In short, we follow the evolution of the $100 \mathrm{pc}$ scale circum-stellar medium of a group of three massive stars $(25$, 32 and $60 M_{\odot}$ ) for the entire evolution of these stars, including the final supernova-explosion (at 8.6, 7.0 and 4.6 Myr, respectively), and a few Myr beyond. We take into account radiative cooling and heating, but no non-thermal processes. The individual bubbles merge and form a superbubble at around $1 \mathrm{Myr}$ of simulation time. This superbubble features a cool shell, which dissipates most of the energy radiatively, and a hot bubble interior at sufficiently high temperature to emit thermal X-ray radiation. We varied the spatial configuration of the three massive stars (compare Table 1) from cospatial (3S0) over tens of parsecs apart $(3 \mathrm{~S} 1,3 \mathrm{~S} 2)$, to very large distances, realised by having each star in a separate simulation (S25, S32, and S60) and adding the result. We performed all runs at a finest resolution of $2 \mathrm{pc}$, and 
Table 1. Simulation parameters.

\begin{tabular}{lcccc}
\hline \hline Label $^{a}$ & Star mass $/ M_{\odot}$ & $X^{b} / \mathrm{pc}$ & $Y / \mathrm{pc}$ & $Z / \mathrm{pc}$ \\
\hline S25 & 25 & 0 & 0 & 0 \\
S32 & 32 & 0 & 0 & 0 \\
S60 & 60 & 0 & 0 & 0 \\
3S0 & 25 & 0 & 0 & 0 \\
& 32 & 0 & 0 & 0 \\
& 60 & 0 & 0 & 0 \\
3S1 & 25 & -30 & 10 & 10 \\
& 32 & -25 & -10 & 0 \\
& 60 & 0 & 0 & 0 \\
3S2 & 25 & -60 & 20 & 10 \\
& 32 & 50 & -10 & 0 \\
& 60 & 0 & 0 & 0 \\
\hline
\end{tabular}

Notes. ${ }^{(a)}$ Label of respective run. Without addition, the run labels refer to the $2 \mathrm{pc}$ resolution runs. Run $3 \mathrm{~S} 1$ has also been carried out at $1 \mathrm{pc}$ resolution, which is denoted by the extension "-mr". Runs 3S0 and $3 \mathrm{~S} 1$ have also been carried out at $0.5 \mathrm{pc}$ resolution, denoted by the extension "-hr". ${ }^{(b)} X, Y$, and $Z$ denote the position of the stars.

repeated two $(3 \mathrm{~S} 0,3 \mathrm{~S} 1)$ at higher resolution, up to $0.5 \mathrm{pc}$. The resolution comparison shows that the X-ray properties are well converged in the X-ray-bright phases. Details are discussed in Appendix A.

We followed Breitschwerdt \& de Avillez (2006) in assuming that resolved hydrodynamic instabilities are the main driver of mixing within the bubbles, and consequently neglected any evaporation due to thermal conduction. Our analysis therefore excludes the main-sequence phase of the most massive star, where the wind is too steady for prominent instabilities to develop. X-ray spectra at the relevant temperatures (one to a few million Kelvin) are dominated by a large number of emission lines. We used a model for emission from tenuous hot plasma which includes the relevant atomic shell physics. Such a model, called "Mekal" (Mewe et al. 1985, 1986; Liedahl et al. $1995)$, is conveniently provided via the XSPEC-package v12.8.0 ${ }^{1}$, (Arnaud 1996; Dorman \& Arnaud 2001). We produced spectra for gas at solar abundances and with temperatures between 0.1 and $10 \mathrm{keV}$ in steps of $0.1 \mathrm{keV}$, and convolved the spectra with the temperature distribution of the hydrodynamic simulations.

\section{Results}

\subsection{X-ray emission in different evolutionary phases}

The contribution to the different parts of the X-ray spectrum depends on the exact temperature of the gas. We show the temporal evolution of the column density of gas in four different temperatures ranges for the representative run 3S1-hr (Table 1 for details) in Fig. 1. For each phase, the synthetic X-ray luminosity is provided in representative bands in Table 2. The maps differ markedly in the different evolutionary phases of the superbubbles: as discussed in Sect. 2, we do not analyse the phase when all three stars are still on the main sequence. In the first Wolf-Rayet phase (Fig. 1, first column), the shell is accelerated, and strong Rayleigh-Taylor instabilities lead to an extended mixing layer (Fig. 2) at temperatures suitable for soft X-ray emission. The luminosity is highest at the low-energies $(<1 \mathrm{keV}$, $>10^{34} \mathrm{erg} \mathrm{s}^{-1}$ ). After merging of the individual wind shells, the debris of the shell interfaces is being pushed into the lower pressure regions, i.e. the bubbles of the less massive stars. The eroded

\footnotetext{
1 http://xspec.gsfc.nasa.gov/
}

shell fragments mix with the gas that pushes them, which again leads to a substantial mass of sub-keV gas. For the main bubble, the amount of sub-keV gas is larger towards the edge of the bubble, as the mixing layer is located outwards of about $25 \mathrm{pc}$ in this phase (compare Fig. 2). The network produced by the Vishniac instability features prominently, as mixed, warm gas is focused in the small cavities in the shell (compare Fig. 3 in Paper I). The $1-2 \mathrm{keV}$ emission is produced almost exclusively in the mixing zone, inside of the sub-keV emission. The unmixed Wolf-Rayet wind itself fills the inner parts of the bubble with hot gas at temperatures between 2 and $4 \mathrm{keV}$. The total emission is however $<10^{32} \mathrm{erg} \mathrm{s}^{-1}$ in the $2-4.5 \mathrm{keV}$ band, and therefore hardly expected to be detected in observations. We find almost no gas above $4 \mathrm{keV}$. Note, that we do not treat cosmic ray acceleration, which would be expected in such conditions, and which may produce non-thermal emission at a few keV (compare e.g. discussion in Yamaguchi et al. 2010).

The second column in Fig. 1 shows the situation $1600 \mathrm{yr}$ after the first supernova explosion. The supernova has produced a substantial amount of high-temperature gas which has boosted the emission in the high-energy bands by about an order of magnitude (compare second row in Table 2). There is no change in the distribution of the colder gas, because the shock wave has not yet reached the higher density regions, and consequently, there is no change to the low-energy bands.

About 14000 yr later (third column in Fig. 1), the shock wave has traversed the entire mixing layer. Because the density increases outwards (Fig. 2) the shock slows down, with less heating in the outer regions. Consequently, the emission spectrum becomes softer. The overall emission, in particular the sub-keV band, has increased by more than a factor of 40 compared to times before the supernova (third row in Table 2).

The X-ray emission fades on a timescale of several $10^{5}-10^{6} \mathrm{yr}$ after each supernova, faster in the higher energy bands. This is due to enhanced mixing: when the shock wave reaches the shell, strong Rayleigh-Taylor instabilities are triggered, mixing entrained gas with the hot gas, thereby reducing its temperature. The acceleration of the shell also leads to some adiabatic-expansion losses, which reduce the X-ray luminosity. We show the superbubble 0.6 Myr after the third explosion in the right column of Fig. 1, where the X-ray emission has faded below earlier phases (fourth row in Table 2). This supernova exploded off-centre and produced a global oscillation of the gas in the bubble interior. At the time shown, the left part is expanded and hence colder, whereas the right part is compressed and therefore hotter. There is no gas at temperatures above $4 \mathrm{keV}$ at this time.

\subsection{Spectral evolution}

Spectra for the entire superbubble of run $3 \mathrm{~S} 1-\mathrm{hr}$ are shown in Fig. 3. At no time, we find a strong cutoff, as would be the case if one temperature would strongly dominate the emission. Instead, the contributions of regions with different temperatures add up to a shape that comes close to a broken power law. Up to $0.5 \mathrm{keV}$, fitting gives a power law index close to -1.5 . For the energy range $0.5-20 \mathrm{keV}$, the fitted power law indices are between -4.4 and -2.7. Discrepancies from single temperature spectra in collisional ionisation equilibrium are also found in observations (Jaskot et al. 2011; Sasaki et al. 2011; Kavanagh et al. 2012). At the given temperatures, departures from collisional ionisation equilibrium are small (Sutherland \& Dopita 1993). Hence, a multi-temperature interpretation of the observations is very probable in agreement with our findings. 


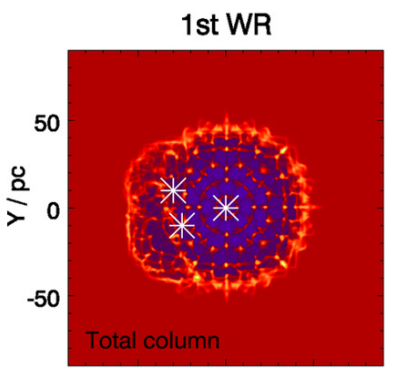

$\begin{array}{lll}-50 & 0 & 50\end{array}$
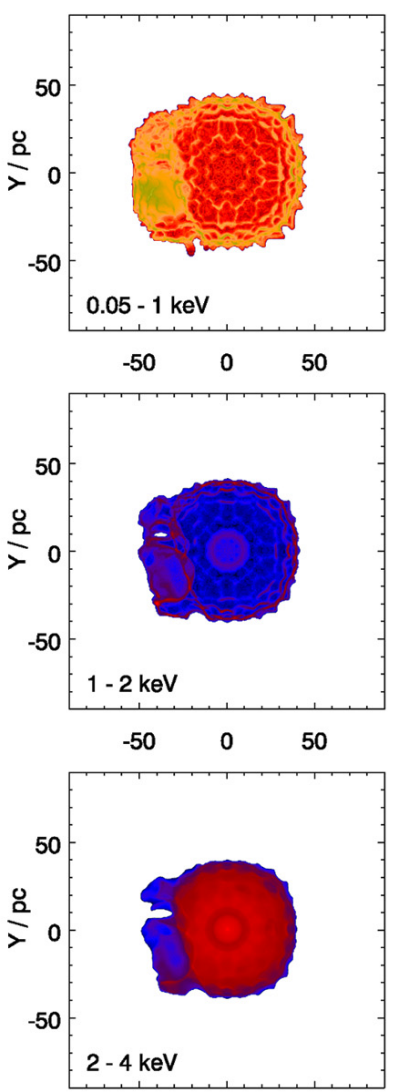

$\begin{array}{lll}-50 & 0 & 50\end{array}$

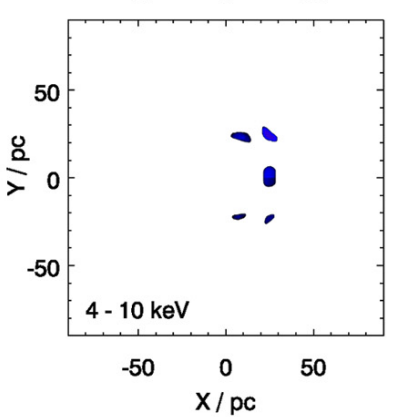

$1 s t S N+1600 \mathrm{yr}$

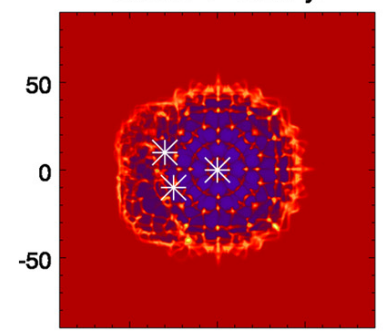

$\begin{array}{lll}-50 & 0 & 50\end{array}$

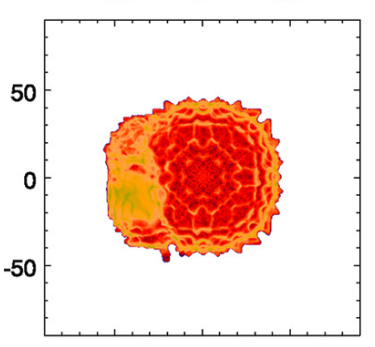

$-50 \quad 50$

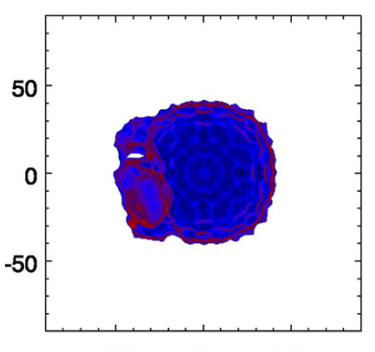

$\begin{array}{lll}-50 & 0 & 50\end{array}$

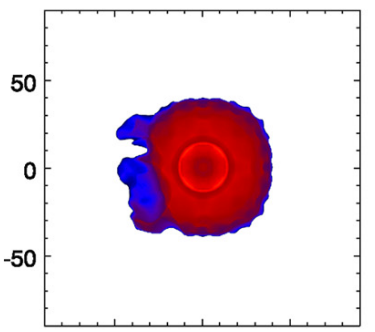

$\begin{array}{lll}-50 & 0 & 50\end{array}$

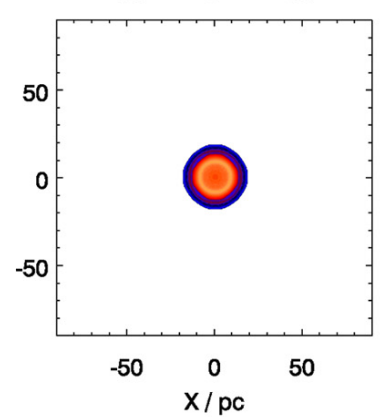

1st X-ray max

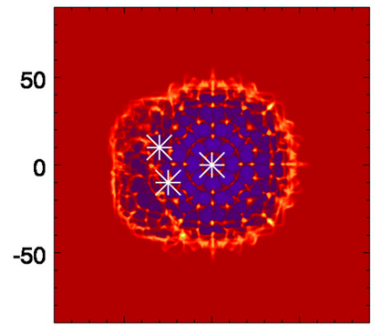

$\begin{array}{lll}-50 & 0 & 50\end{array}$

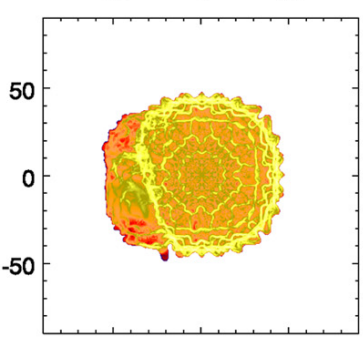

$\begin{array}{lll}-50 & 0 & 50\end{array}$

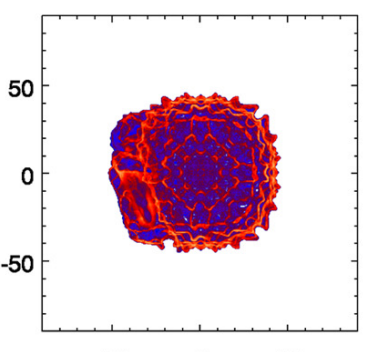

$\begin{array}{lll}-50 & 0 & 50\end{array}$

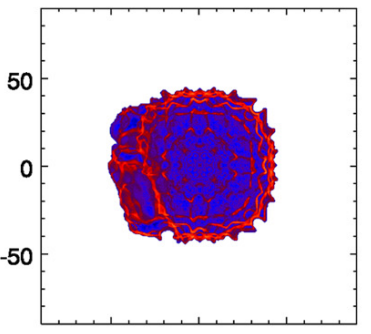

$\begin{array}{lll}-50 & 0 & 50\end{array}$

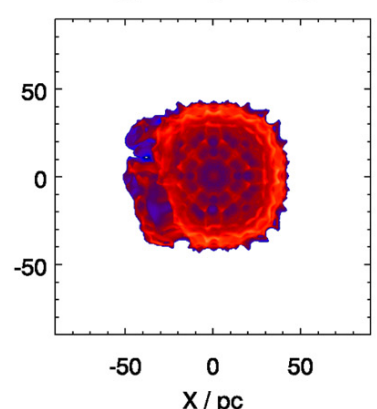

Fading superbubble

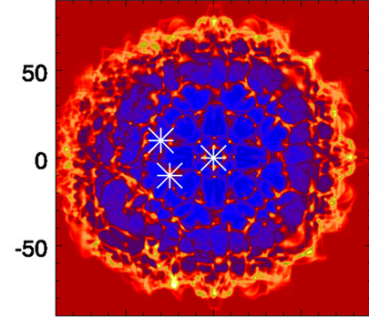

$\begin{array}{lll}-50 & 0 & 50\end{array}$

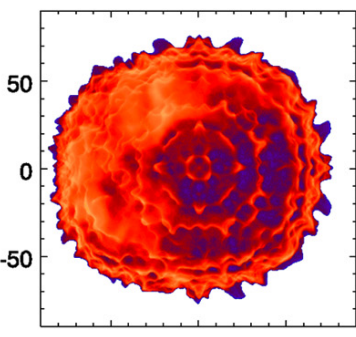

$\begin{array}{lll}-50 & 0 & 50\end{array}$

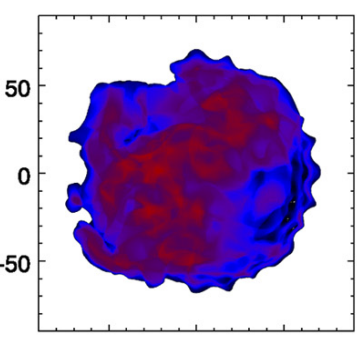

$\begin{array}{lll}-50 & 0 & 50\end{array}$

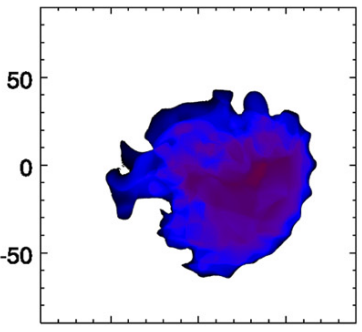

$\begin{array}{lll}-50 & 0 & 50\end{array}$

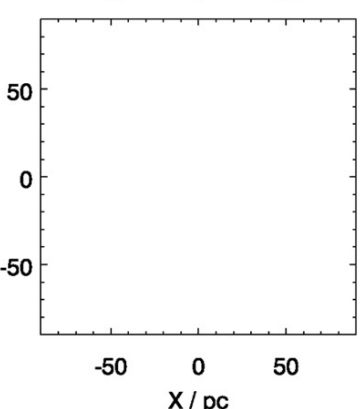

$\log \left(X\right.$-ray column $/ \mathrm{g} \mathrm{cm}^{-2}$ )

Fig. 1. Time evolution (from left to right; the snapshot time is indicated on the top of each column) of X-ray properties of simulation 3S1-hr, featuring 3 massive stars at about 30 pc distance from each other. From top to bottom, the plots show column density distributions of all gas (the three massive stars are indicated as white stars), and of X-ray-emitting gas at temperatures of $0.05-1 \mathrm{keV}, 1-2 \mathrm{keV}, 2-4 \mathrm{keV}$ and $4-10 \mathrm{keV}$. The colour bar gives values relevant to the X-ray panels, but the colour scale is the same for the total gas column. The left column shows the superbubble in the Wolf-Rayet phase of the central, most massive star. The middle columns display two different snapshots shortly after the first supernova, first with prominent high energy and then with very strong soft emission, when the shock approaches the shell. Right column: $0.6 \mathrm{Myr}$ after the final supernova, which occurred off-centre, the hot gas performs global oscillations, which leads to the different morphologies in the different bands. See Table 2 for exact snapshot times and corresponding X-ray luminosities. (Online movie.) 
M. Krause et al.: Emergence of superbubbles - X-ray properties

Table 2. X-ray luminosities at different characteristic times and energy bands.

\begin{tabular}{lcccccccc}
\hline \hline Time/Myr & Description & \multicolumn{7}{c}{ Log (luminosity/(erg s-1)) } \\
& & $0.1-20 \mathrm{keV}$ & $0.2-12 \mathrm{keV}$ & $0.2-0.5 \mathrm{keV}$ & $0.5-1 \mathrm{keV}$ & $1-2 \mathrm{keV}$ & $2-4.5 \mathrm{keV}$ & $4.5-12 \mathrm{keV}$ \\
\hline 4.5251 & First WR & 34.47 & 34.27 & 34.11 & 33.71 & 32.61 & 31.78 \\
4.6016 & First SN & 34.36 & 34.17 & 33.99 & 33.59 & 32.67 & 32.46 & 32.43 \\
4.6159 & First X-ray max. & 36.12 & 35.93 & 35.77 & 35.38 & 34.32 & 33.30 & 32.72 \\
9.2236 & Fading superbubble & 33.71 & 33.53 & 33.34 & 33.02 & 32.18 & 31.34 & 30.56 \\
\hline
\end{tabular}

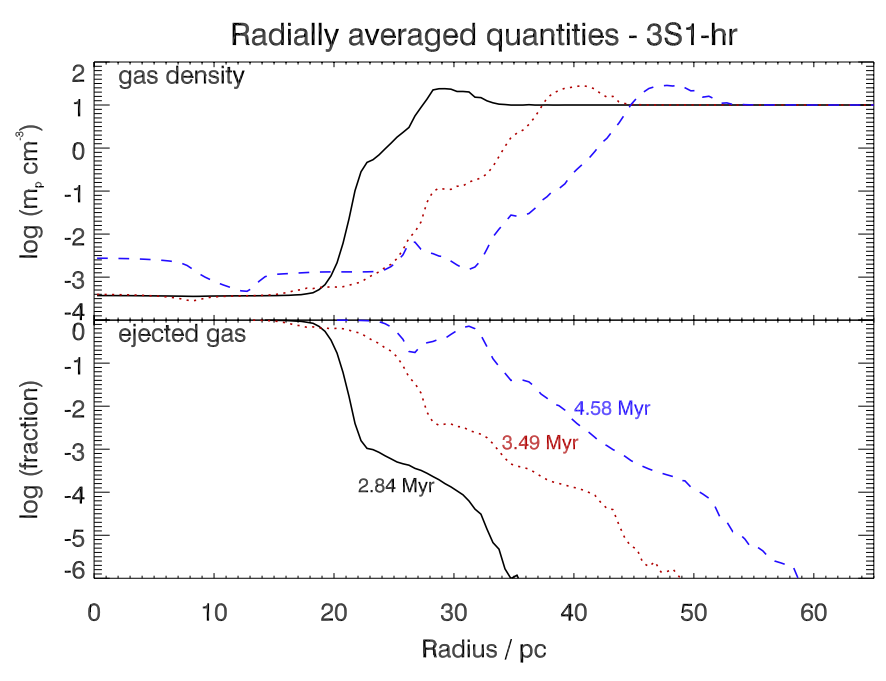

Fig. 2. Density (top) and fraction of ejected gas as a function of distance from the most massive star (radius) for three different times (solid black: 2.84 Myr, dotted red: $3.49 \mathrm{Myr}$, dashed blue: $4.58 \mathrm{Myr}$ ), averaged at constant radius. In the main-sequence phase of the $60 M_{\odot}$ star $(2.84 \mathrm{Myr})$, there is a sharp contact surface at $20 \mathrm{pc}$, where the ejected gas fraction suddenly drops to $10^{-3}$. During the Wolf-Rayet phase, the wind power increases. Rayleigh-Taylor instabilities at the accelerating shell create an extended mixing layer. $20000 \mathrm{yr}$ before the supernova of the $60 M_{\odot}$ star (dashed blue curves), the mixing layer extends between about 27 and $40 \mathrm{pc}$, if defined by ejected gas fractions between 1 and 10 percent.

\subsection{Spatial configuration of the massive stars}

We investigated different configurations of the stars, varying the distance from co-spatial over two configurations with tens of parsecs distance to very large distances (separate simulations for each star). For this comparison, we took only simulations with a resolution of 2 pc. The X-ray luminosity, especially the hard bands, fades faster, as the stellar distances increase (Fig. 4). This is, because we approach the isolated bubbles case: a supernova exploding in a smaller bubble produces a higher pressure, hence a stronger shell acceleration and thus more mixing via the Rayleigh-Taylor instability and faster adiabatic losses.

We defined two colour indices as the ratio of the emitted power in bands as defined in Table 2:

$C 1=\log _{10}([1-2 \mathrm{keV}] /[0.2-1 \mathrm{keV}])$

$C 2=\log _{10}([4.5-12 \mathrm{keV}] /[1-2 \mathrm{keV}])$.

For the strong clustering runs, both $C 1$ and $C 2$ have values close to -1.7 throughout the evolution, except shortly after each supernova. As we placed the stars further away from each other, both $C 1$ and $C 2$ dropped, sometimes substantially.

The X-ray luminosity directly associated with the supernova explosions, until a few $10^{5} \mathrm{yr}$ after the explosion, varies remarkably little in the different bands. Even the final supernova produces the same peak luminosity in all bands, irrespective of the supernova exploding in its own wind bubble or the cavity being shaped by the complete evolution of two more massive stars at different positions. We find a peak X-ray luminosity of roughly $10^{36} \mathrm{erg} \mathrm{s}^{-1}$ after each supernova for all our configurations.

The increase in the colour indices after each supernova explosion is significant and the decay back to the base level is well resolved in time: $C 1$ increases to up to -0.5 and $C 2$ to positive values.

In summary, unless a supernova has just exploded, clustering sometimes leads to higher X-ray luminosities in all bands and harder colour indices. When a supernova actually explodes, the X-ray luminosity is always increased to the same level in all bands.

\subsection{Total energy emitted in $X$-rays}

The time-integrated radiated energy as a fraction of the current cumulative input energy is shown in Fig. 5. For each energy band, the emission stays at the same value within an order of magnitude, throughout the evolution. The ratio between energy bands is almost constant. The curves decrease after the first supernova, but quickly return to a similar level, as the injected energy is emitted when the shock reaches the shell. The curves then stay constant until shortly before the next supernova. Generally, the emission drops by a factor of a few between the $0.2-0.5$ and the $0.5-1 \mathrm{keV}$ bands, a further factor of ten for the $1-2 \mathrm{keV}$ band, and another factor of ten to the higher energy bands. The total emitted energy in X-rays is a few times $10^{-4}$ of the injected energy. This result can be used to predict the total X-ray luminosity of nearby galaxies from such superbubbles (Sect. 4.3, below).

\section{Comparison to observations}

\subsection{General $X$-ray properties of superbubbles}

Dunne et al. (2001) present a ROSAT study of 13 superbubbles in the LMC. The superbubbles have diameters of about $100 \mathrm{pc}$, very similar to our simulations. They find diffuse X-ray emitting regions in each case, with a soft spectrum and a patchy morphology, sometimes outside apparent shells as defined by $\mathrm{H} \alpha$. They report correlations of the X-ray luminosity with the $\mathrm{H} \alpha$ luminosity, the expansion velocity of the shells, and the OB star count. Apart from the correlation with the OB star count (which we do not address here, because all our simulations have the same three stars) all these findings are well explained by our simulations: whenever two bubbles merge, the pressure in each individual bubble is likely very different. In our simulations (Fig. 1), the emission is enhanced in the gas flooding the low-pressure bubble, because the bubble interface is eroded. Such gas should therefore be highlighted in observations. We find the gas to become X-ray bright, when entrained and mixing shell gas is 


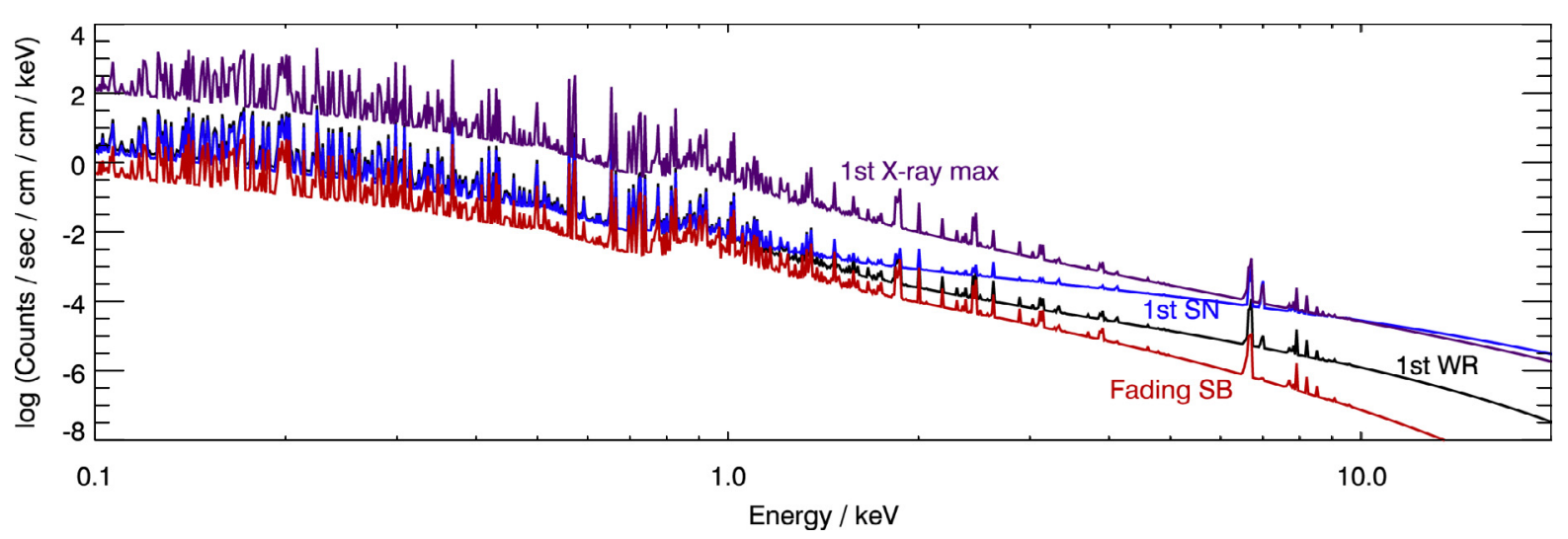

Fig. 3. Thermal X-ray spectra for run 3S1-hr. The time sequence of the labels is 1st WR (black), 1st SN (blue), 1st X-ray-max (violet), and fading SB (red). They refer to the descriptions in Table 2. A supernova inside a superbubble first increases the hard X-rays and then the softer parts by up to two orders of magnitude compared to immediately before the explosion. All the spectra reflect the multi-temperature structure.

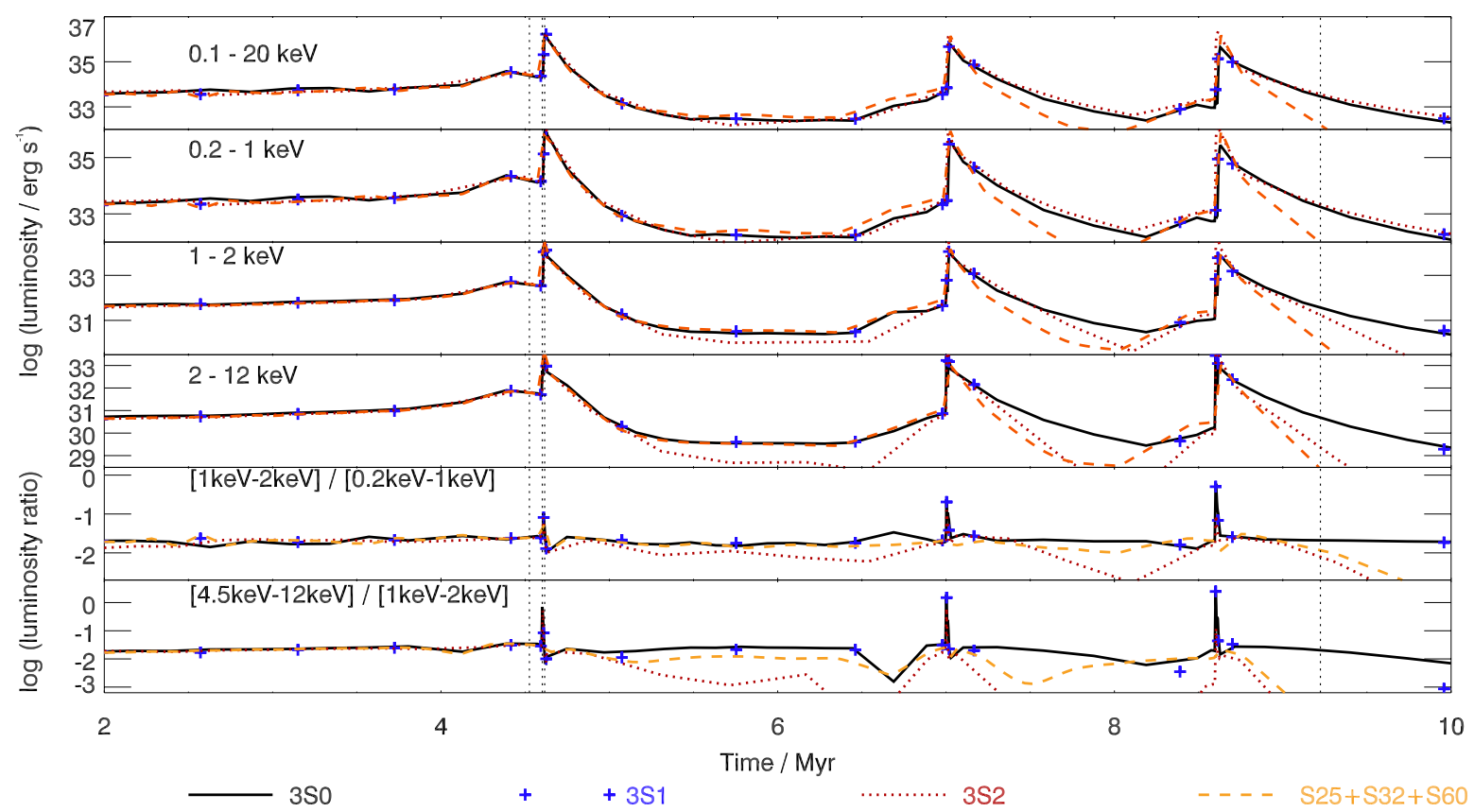

Fig. 4. Integrated thermal X-ray luminosity for four energy bands (four top panels, energy bands indicated in the respective panel) and two X-ray colour indices (bottom panels) as a function of time since the coeval formation of the three massive stars. The configurations of the 3 massive stars are: all stars at the same place (3S0, solid black), as indicated in Fig. 1 (3S1, blue pluses), at a significantly larger distance (3S2, red dotted) and all stars in separate simulations (dashed orange line). The vertical dotted lines indicate the snapshot times used for Fig. 1. All runs show a strong variability in all X-ray bands. Clustering increases the X-ray output long after a supernova event (peaks) and hardens the spectrum. Both colour indices stay close to -1.7 for most of the time. See text for more details.

shocked due to a supernova. This is necessarily connected to an acceleration of the shell, in line with the observed correlation between X-ray luminosity and expansion velocity. The enhanced expansion velocity then boosts the $\mathrm{H} \alpha$ luminosity at the leading shock.

Zhekov \& Park (2011) present a Suzaku study of the Wolf-Rayet wind bubble NGC 6888. They find emission from a variety of temperatures with a dominant low-temperature component, but also contributions from gas above $1 \mathrm{keV}$. The emission is limb-enhanced and originates in clumps. This agrees very well with our simulations, which show prominent RayleighTaylor instabilities in the Wolf-Rayet phase entraining filaments from the shell into the bubble. The X-rays would then be produced in the mixing region.
We compare luminosities and emission-measure weighted temperatures from our high-resolution simulations to these observational data in Fig. 6 (the tracks of the other ones are similar). Here, we set the X-ray luminosity to zero below $10^{33.5} \mathrm{erg} \mathrm{s}^{-1}$, because for lower luminosities we expect either to be dominated by heat conduction effects (main sequence phase), which are not included in the simulations, or the simulations are not numerically converged (compare Fig. A.1). The plot shows that the values we derive are generally in the range of expectations. Our simulation results cover the full range of observed bubble temperatures. Depending on the assumptions about absorption in the observations, they also cover part or most of the range of observed luminosities. In detail, the LMC superbubbles seem to require somewhat hotter and at the same time 
M. Krause et al.: Emergence of superbubbles - X-ray properties

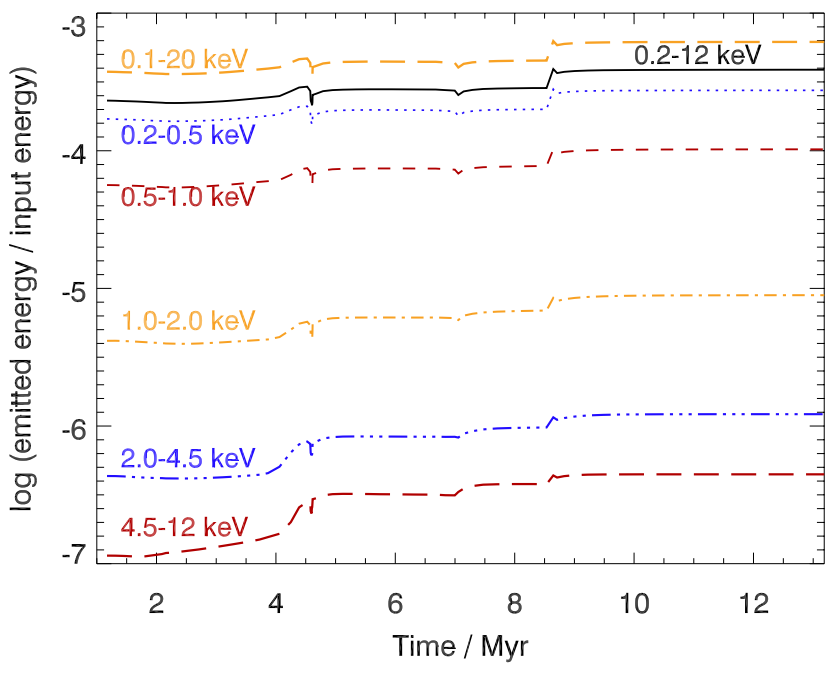

Fig. 5. Cumulative energy output as fraction of the cumulative energy input over time for various energy bands indicated next to the respective curves and for run 3S1-hr, shown in Fig. 1.

more luminous gas than what we find in our simulations. Also, the duration of the X-ray luminous phase of order $10^{5} \mathrm{yr}$ (Fig. 4) appears short. It would mean that only few superbubbles are $\mathrm{X}$-ray luminous. While we are not aware of a study that analyses the X-ray luminosities of an e.g. optically selected parent sample, this number appears to be small.

For the Wolf-Rayet winds, we find lower luminosities $\left(\approx 10^{34} \mathrm{erg} \mathrm{s}^{-1}\right)$ than for superbubbles. This agrees well with observations (also Fig. 6).

\subsection{The Orion-Eridanus bubble}

The Orion-Eridanus cavity (e.g. Brown et al. 1995; Welsh et al. 2005; Voss et al. 2010; Jo et al. 2012, Figs. 7 and 8) is a very nearby superbubble, only a few hundred parsecs away. It extends about 40 degree on the sky. It was discovered as a prominent source in the ROSAT all-sky survey (Snowden et al. 1997; Freyberg \& Egger 1999). ROSAT continues to be the best source of soft X-ray data for such an extended object. An analysis of the available XMM-Newton data (Lubos 2012) showed that more observing time and dedicated pointings avoiding sources would be required to measure the extended X-ray emission. The data have not yet been evaluated separately for the Orion-Eridanus cavity. We show the ROSAT images in two energy bands in Fig. 7. An $\mathrm{H} \alpha$ map of the same region is shown in Fig. 8. The $1 / 4 \mathrm{keV}$ emission is bounded by $\mathrm{H} \alpha$ emission away from the Galactic plane (downwards in Figs. 7 and 8). It fades towards the galactic plane as the higher energy emission becomes more prominent. This is very similar to our simulated morphologies at late times (compare Fig. 1). In the simulations, the effect is due to global oscillations of the hot gas in the superbubble. The Orion-Eridanus cavity has had enough time for such global oscillations to establish: from the stellar population, one expects about one supernova every Myr (Voss et al. 2010). The sound crossing time is about $0.5 \mathrm{Myr}\left(150 \mathrm{pc}, c_{\mathrm{s}}=300 \mathrm{~km} \mathrm{~s}^{-1}\right)$. This would suggest a significant probability to capture the superbubble in the second or third period of a global oscillation. While a precise derivation of the luminosity of the Orion-Eridanus superbubble has turned out to be difficult due to its proximity and spatially varying absorption, it is likely that it significantly exceeds the $10^{34} \mathrm{erg} \mathrm{s}^{-1}$ (estimated from the counts in Fig. 7) we typically measure for this phase.

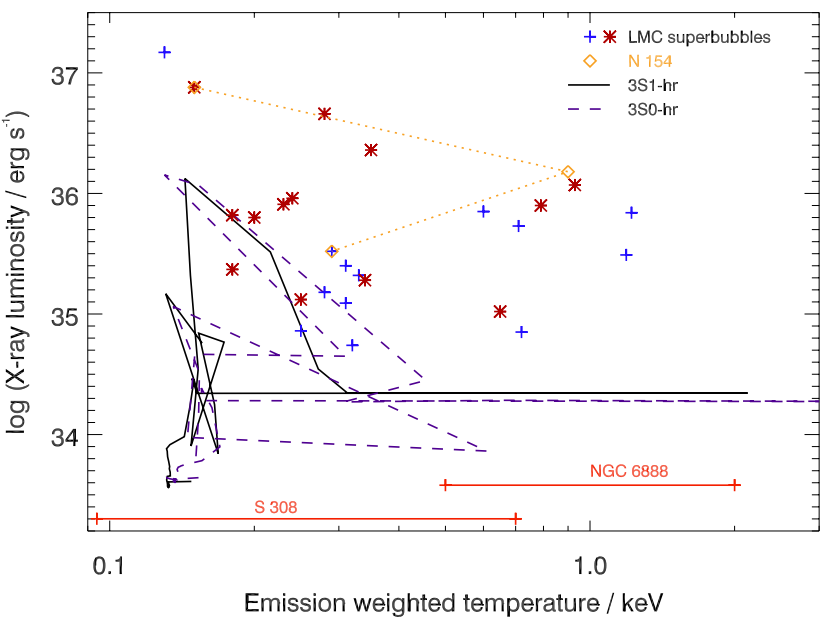

Fig. 6. Luminosity-temperature diagram for run 3S1-hr (solid black line), shown in Fig. 1, and for run 3S0-hr, where the massive stars are all co-spatial. For this comparison, the X-ray luminosity is integrated between 0.1 and $2.4 \mathrm{keV}$ corresponding to the ROSAT PSPC instrument. The temperature is the average temperature of the superbubble weighted by the emission measure. This is compared to the data of a sample of LMC superbubbles observed with ROSAT by Dunne et al. (2001, red stars and the blue pluses correspond to the same sample but different absorption corrections). The light-orange diamonds connected by the dotted line correspond to different measurements for the bubble N 154 in the LMC. The two lower temperature measurements are from ROSAT. The higher temperature measurement is from Sasaki et al. (2011) with $X M M$-Newton. This gives an idea of the observational uncertainty. We also show data for the two X-ray-detected Wolf-Rayet bubbles, S308 (Toalá et al. 2012) and NGC 6888 (Zhekov \& Park 2011).

\subsection{Diffuse $X$-ray luminosity of galaxies}

The soft $(<2 \mathrm{keV})$ as well as the hard $(>2 \mathrm{keV}) \mathrm{X}$-ray emission correlates with tracers of the SFR (Persic et al. 2004; Strickland et al. 2004b) ${ }^{2}$. Our spectra (Fig. 3) and the cumulative radiated energy plots (Fig. 5) make clear that superbubbles do not contribute much - thermally - to the hard band, and indeed the observed correlation in the hard band is thought to be due to the emission of high-mass X-ray binaries (neutron stars with massive OB companions, Persic et al. 2004). Here, we assess the contribution of superbubbles to the soft X-ray emission of galaxies.

As shown above, a superbubble experiences strong changes in its diffuse X-ray luminosity over time. For an entire galaxy, we can assume that we see a large number of superbubbles in uncorrelated evolutionary states. The ensemble average over all superbubbles in the galaxy would then be equal to the time average of the emission of one superbubble scaled by the galaxy's SFR. Above (Sect. 3 and Fig. 5), we found for the conversion efficiency $f_{\text {sX }}$ from mechanical power to diffuse soft X-ray luminosity a factor of a few times $10^{-4}$. One supernova occurs approximately for every $100 M_{\odot}$ of stars formed (e.g. Dahlen et al. 2012). There is a roughly equal energetic contribution from winds and supernovae, respectively, of $10^{51}$ erg averaged over the population (Voss et al. 2009), for each massive star.

\footnotetext{
2 Franceschini et al. (2003) do not find a correlation between the starformation rate and the thermal X-ray emission. However, from our spectra, we caution that due to the multi-temperature nature of superbubbles, it may be difficult to properly distinguish thermal and non-thermal components from the observed spectra. Also, they only cover one dex in $\mathrm{X}$-ray luminosity.
} 

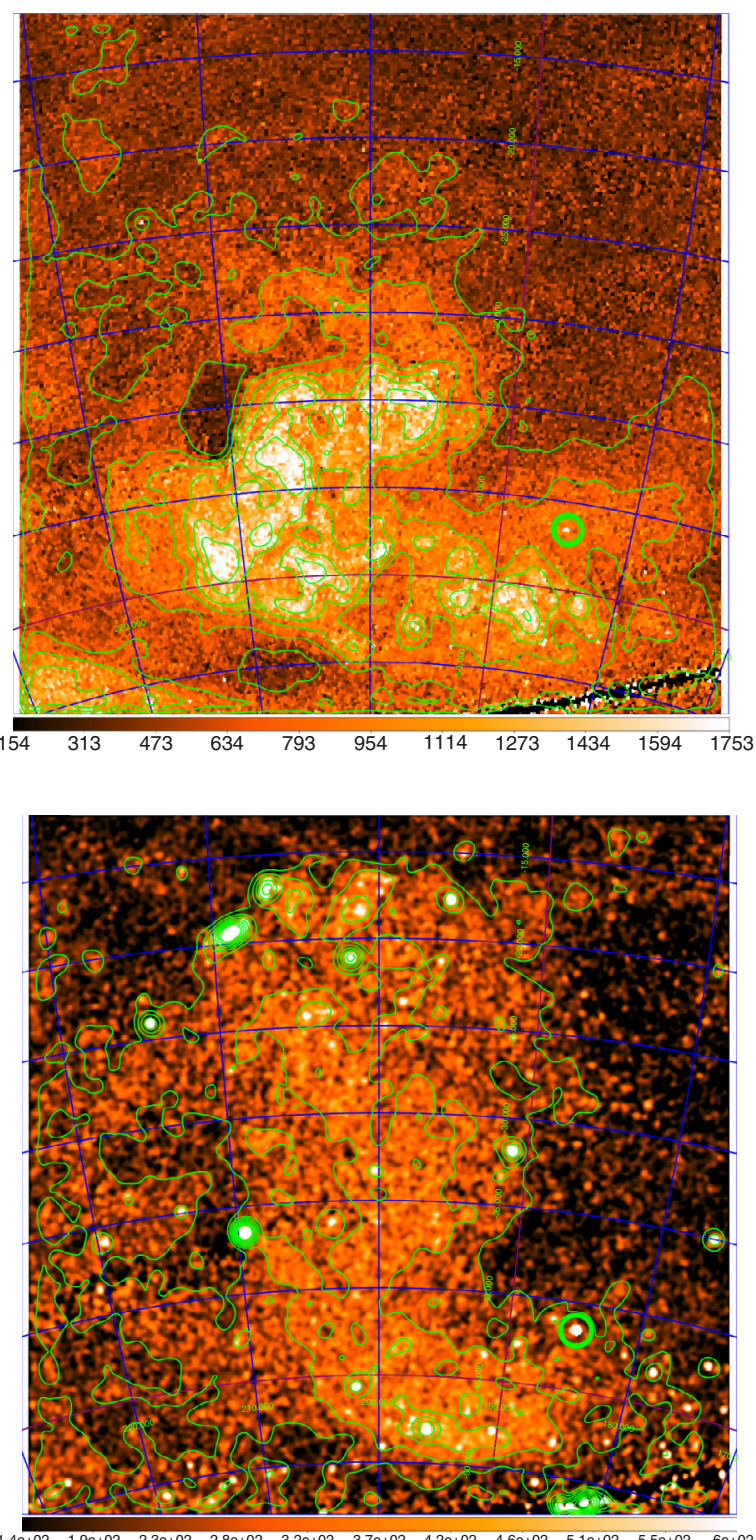

Fig. 7. X-ray maps of the Orion-Eridanus superbubble (same region as in Fig. 8) in the 0.1-0.4 keV (top, contour levels: 700, 900, 1100, 1300, 1500,1900 ) and the $0.5-2 \mathrm{keV}$ (bottom, contour levels: 220, 300, 380, $460,540,620,700$ ) bands from the ROSAT all-sky survey (Snowden et al. 1997). The colour scale units are $10^{-6}$ counts $\operatorname{arcmin}^{-2} \mathrm{~s}^{-1}$. The scale is linear and does not start at zero in order to de-emphasise unrelated background. The X-ray emission of the superbubble interior is delineated by shells seen in $\mathrm{H} \alpha$ (compare Fig. 8). The softer and harder $\mathrm{X}$-ray bands emphasise gas at different temperature. We propose that this may relate to a global oscillation of the superbubble, similar as seen in the simulations.

These assumptions lead to a prediction for the average diffuse soft X-ray output of of star-forming galaxies of

$L_{\mathrm{SX}} \approx \frac{2 \times 10^{51} \mathrm{erg}}{100 M_{\odot}} f_{\mathrm{sX}} S F R=10^{38} \mathrm{erg} \mathrm{s}^{-1} \frac{S F R}{M_{\odot} \mathrm{yr}^{-1}}$

from our simulations (0.2-2 keV band).

We compare this to observations of nearby galaxies in Fig. 9. The diffuse X-ray luminosity in these galaxies correlates with the star-formation rate, as expected, if the X-ray emission is caused by a process related to star formation. The ROSAT value

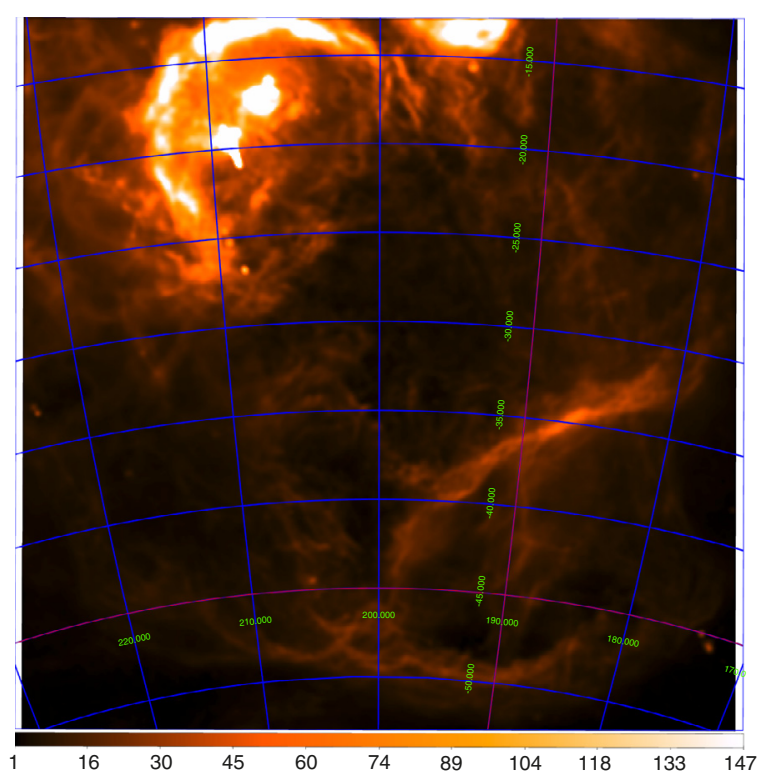

Fig. 8. H $\alpha$ map of the Orion-Eridanus superbubble (Reynolds \& Ogden 1979, same region as in Fig. 7). The colour scale is linear and in units of Rayleigh $\left(4 \pi \times 10^{-4}\right.$ photons $\left.\mathrm{cm}^{-2} \mathrm{~s}^{-1} \mathrm{sr}^{-1}\right)$. A galactic coordinate system is indicated with degree labels in green. The projection is areaconserving and centred on the Galactic anti-centre. The two bright regions near $(l, b)=(-208,-18)$ are the Orion clouds. The bright arc towards the top left of the Orion clouds is Barnard's loop, probably a shell due energy injection from the Orion clouds. It delineates the extent of the superbubble towards the Galactic plane. The arc-like features towards the bottom of the map may also be related to the shell and delimit the X-ray emission (compare Fig. 7). The shell is also traced in HI (Brown et al. 1995).

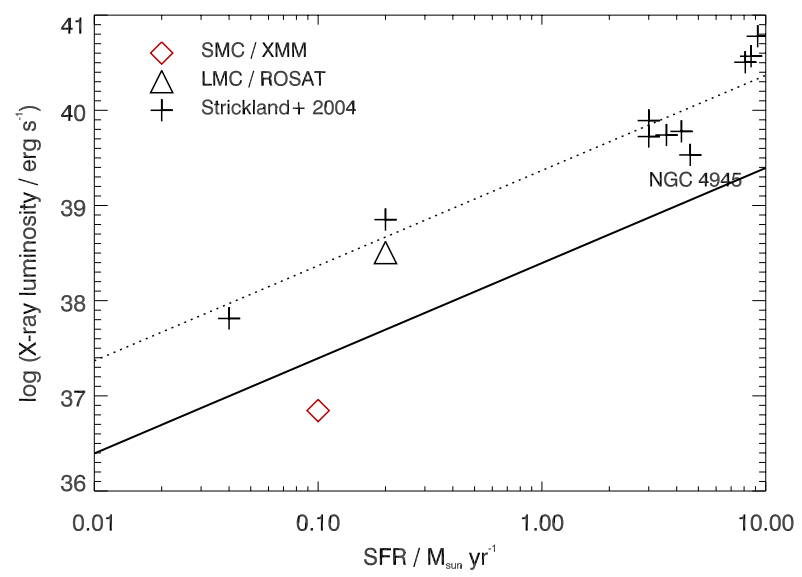

Fig. 9. Diffuse X-ray luminosity in the $0.2-2 \mathrm{keV}$ band versus star formation rate in nearby galaxies. Black plus signs are data from Strickland et al. (2004a) for a sample of nearby galaxies with a large range of star-formation properties. The black triangle shows the ROSAT measurement of the LMC form Sasaki et al. (2002). The result from XMM-Newton survey of the SMC (Sturm 2012; Sturm et al. 2013) is indicated by the red diamond. The dotted line is a fit to the Strickland et al. data. The source which is furthest below this line (NGC 4945) is marked. The solid line is the prediction from our analysis for solar metallicity. It is below the fit line for the Strickland et al. data by a factor of 9 , which would decrease to 6 if the $0.1-0.2 \mathrm{keV}$ band would be added. For the SMC metallicity, the prediction would be lower by a factor of ten. See Sect. 5 for possible explanations for the discrepancy.

for the LMC is close to the fit line to the Chandra sample of Strickland et al. (2004a), whereas the X-ray luminosity of the 
Small Magellanic Cloud (SMC) is a factor of 30 lower. One reason for this is that the proximity of the SMC together with the spectral resolution capabilities of the XMM-Newton telescope ensure the best possible background separation. These efforts have lead to a reduction of X-ray luminosity compared to the previous ROSAT measurement of diffuse X-ray luminosity for the SMC (Sasaki et al. 2002) by almost 40 per cent. It is possible that accounting for unresolved background sources would also lead to a reduction of the LMC luminosity. The same may even be true for the luminosities determined with Chandra for the sample of Strickland et al. (2004a), because the better spatial resolution of Chandra is compensated by the larger distances. We note that the source which lies furthest below the fit line, NGC 4945, has the best point source sensitivity within the Chandra sample, $6 \times 10^{36} \mathrm{erg} \mathrm{s}^{-1}$. Another reason why the SMC data point is so much below the correlation defined by the other data points is certainly the low metallicity of the ISM in the SMC of only $1 / 5$ of the solar value (Russell \& Dopita 1992). This affects the total X-ray luminosity by almost 1 dex (Sutherland \& Dopita 1993). Our prediction would hence have to be adjusted about 1 dex downwards, leading to a consistent underprediction of the soft X-ray luminosity in all these galaxies. In the following, we adopt the viewpoint that the reported X-ray luminosities (apart from the SMC result) may perhaps be a factor of two to large, due to unaccounted backgrounds. In this case they would still be significantly above our predicted line.

\section{Discussion}

We predicted the X-ray properties of superbubbles based on 3D hydrodynamics simulations. The essential assumption behind this approach is that the X-ray properties are a consequence of the repeated basic sequence: shock-heating - shell acceleration and hydrodynamic instabilities - mixing.

Several observed properties are reproduced very well by our simulations:

- The X-ray luminosity of superbubbles is extremely variable in space and time. We find variations of two orders of magnitude in total X-ray luminosity. This agrees very well with the observation that some superbubbles are X-ray bright and some are X-ray dim (e.g. Jaskot et al. 2011).

- The peak X-ray luminosity is about $10^{36} \mathrm{erg} / \mathrm{s}$. This a direct consequence of the interplay of shock-heating and large-scale instabilities with subsequent mixing well modelled in our 3D simulations. This agrees well with observations (Fig. 6).

- The predominant temperature of the X-ray emitting material is (for most of the time) sub-keV. This difference compared to expectations from simple analytic bubble models (e.g. Dunne et al. 2001) is due to the enhanced mixing due to Rayleigh-Taylor instabilities triggered by the strong dependence of the kinetic energy input on time.

- Large-scale (in space) spectral changes are expected due to global oscillations inside the bubble. We suggest this is the case in the nearby Orion-Eridanus superbubble.

There are also disagreements with observations:

- The X-ray luminosity seems to decay to fast. We infer this from the large number of X-ray bright superbubbles, and also from the underpredicted X-ray luminosities of nearby galaxies.
- While we cover part of the region in the luminositytemperature diagram (Fig. 6), the temperature of the X-ray emitting gas in observed superbubbles frequently seems to be a factor of a few higher than what we predict.

How can we explain these disagreements?

We believe the disagreement can hardly be related to numerical issues: in the relevant phases, the derived properties are well converged, and it is clear that the fact that the X-ray luminosity is not fully converged in the very low luminosity phases does not have any effect on the X-ray properties of any luminous phase later on (Appendix A and Fig. A.1).

The ambient density might differ from the one we assumed $\left(10 \mathrm{~m}_{\mathrm{p}} \mathrm{cm}^{-3}\right)$. This would change the density of entrained shell filaments and the growth rate of the Rayleigh-Taylor instability, because the shell dynamics is linked to the ambient density. We investigated this briefly with two additional simulations using one $25 M_{\odot}$ star, for which we varied the ambient density by a factor of ten upwards and downwards compared to our standard value. We found a factor of a few higher peak luminosity for the higher ambient density run. However, we believe this should be verified at finer resolution. This issue is beyond the scope of the present work. For N 154, Sasaki et al. (2011) derive an ambient density of $13 \mathrm{~m}_{\mathrm{p} \mathrm{cm}} \mathrm{cm}^{-3}$, quite close to the value we used. Still, they find a temperature and luminosity value significantly outside the region covered by our simulations (Fig. 6).

Observed X-ray-bright superbubbles usually contain more massive stars than the three we assumed. The result of our simulations is that the X-ray bright phases are essentially independent. This should remain true also for richer star clusters, where the supernovae might follow one another more rapidly as long as the X-ray bright phases associated with the individual supernovae do not overlap. This condition should be satisfied if the time span between consecutive explosions exceeds about $1 \mathrm{Myr}$, corresponding to about 30 massive stars powering a given superbubble. It is exceeded in many objects, e.g. N 154 (Sasaki et al. 2011). Moving the luminosity peaks closer together (e.g. Fig. 4) may keep the individual superbubbles close to the peak luminosity, but would hardly affect the cumulative energy emitted in X-rays, and thus the diffuse X-ray luminosities for nearby galaxies (Fig. 9) would still be underpredicted.

These findings might point to an agent which reduces mixing in observed superbubbles after a given shock-heating event: the main reasons for the sharp decline in X-ray luminosity after a maximum are adiabatic expansion and mixing with entrained material (radiation losses are negligible). Less mixing after the passage of the supernova shock wave would therefore keep the luminosity high for a longer period. This would of course also keep the temperature at a higher level, as required. It might also keep the surface brightness during the global oscillations higher, as seems to be required for the case of the Orion Eridanus superbubble.

A magnetic field of significant strength might reduce the mixing via a suppression of instabilities (e.g. Jun et al. 1995). This might happen on large scales, as well as on sub-resolution scales. The magnetic field is likely strong in superbubbles: the expansion of the ejecta will produce some magnetic energy via field line stretching. Subsequent turbulence should then randomise the field components. These mechanisms successfully explain the magnetic fields in the lobes of extragalactic radio sources (Gaibler et al. 2009; Huarte-Espinosa et al. 2011). The ambient material is strongly compressed in the expanding shell. Since much of the internal energy is lost due to radiation, the shell material might actually be supported by the 


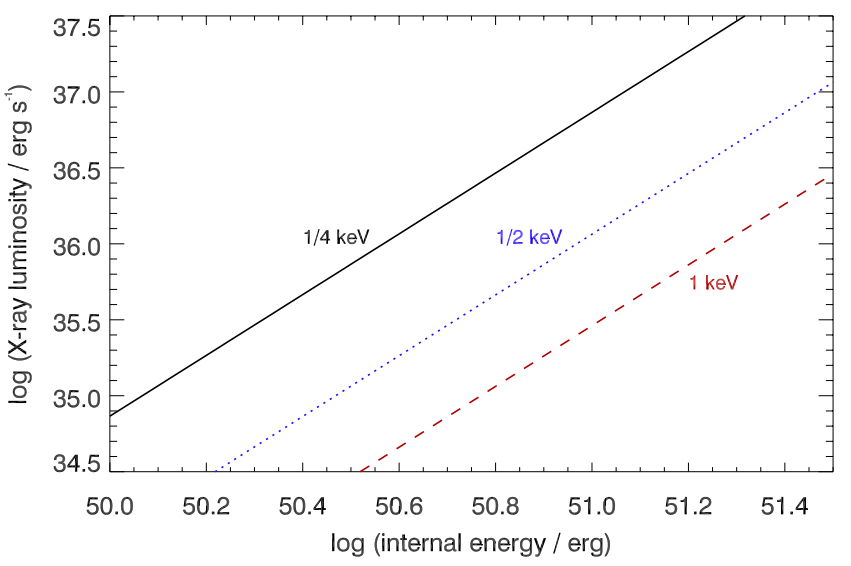

Fig. 10. X-ray luminosity versus total internal energy for simple onezone emission models. See Sect. 5 for details.

magnetic field (Dopita \& Sutherland 1996). Magnetic fields in superbubbles have been observed (Heald 2012). Their effect has also been studied in simulations (e.g. Stil et al. 2009, and references therein), but implications for the thermal X-ray properties have so far not been investigated. However, because mixing is important to produce the intermediate density regions which are responsible for the X-ray emission in the first place, magnetic fields may not entirely suppress the instabilities.

After a short X-ray bright phase, our simulated superbubbles dim in X-rays. At the same time, they also loose their energy due to bubble dynamics: the expansion work on the shell is then lost to (optical) radiation. The typical energy content is $10^{51}$ erg in the X-ray bright phase and $10^{50}$ erg otherwise. The luminosity $L$ is related to the heat energy content $E$ by $L=((\gamma-1) E / k T)^{2} \Lambda / V$, where $\Lambda$ denotes the cooling function, $\gamma=5 / 3$ the adiabatic index and $V$ the volume. For an order of magnitude estimate, let us take $\Lambda$ from the solar metallicity models of Sutherland \& Dopita (1993) in collisional ionisation equilibrium, and set the volume to $V=4 \pi(50 \mathrm{pc})^{3} / 3$. The resulting curves are shown in Fig. 10 for $k T=1 / 4,1 / 2$ and $1 \mathrm{keV}$. The plot demonstrates that with a bubble energy of $10^{51} \mathrm{erg}$, a mixture of 1/4-1 keV plasma can account for the observed X-ray luminosities of $10^{35}-10^{36} \mathrm{erg} \mathrm{s}^{-1}$. With $10^{50} \mathrm{erg}$, this is not possible. This suggests that the dynamical energy loss time in real superbubbles is delayed, probably by a factor of a few, compared to our simulations.

An alternative explanation for the underprediction of the soft $\mathrm{X}$-ray luminosity in galaxies may of course be that other sources contribute also to the observed luminosity. Persic et al. (2004) have suggested that high-mass X-ray binaries may also contribute to the soft X-ray emission. We have noted earlier that point source contamination is indeed an issue for galaxy-scale observations. However, so far the best available observations are still above the luminosity we would predict (compare Sect. 4.3).

Some superbubbles have non-thermal contributions to the $\mathrm{X}$-ray spectra from cosmic rays (compare Sect. 1). Taking 30 Doradus as example, one would expect at most a similar non-thermal X-ray luminosity than the thermal one (Bamba et al. 2004). Because not all superbubbles are detected in X-rays (Yamaguchi et al. 2010), it appears unlikely that a non-thermal contribution could explain the discrepancy.

The microscopic mixing process itself is not treated explicitly in our analysis. We simply assume here that the gas phases are microscopically mixed at the resolution limit. We believe this is justified, because the smaller scale instabilities responsible for
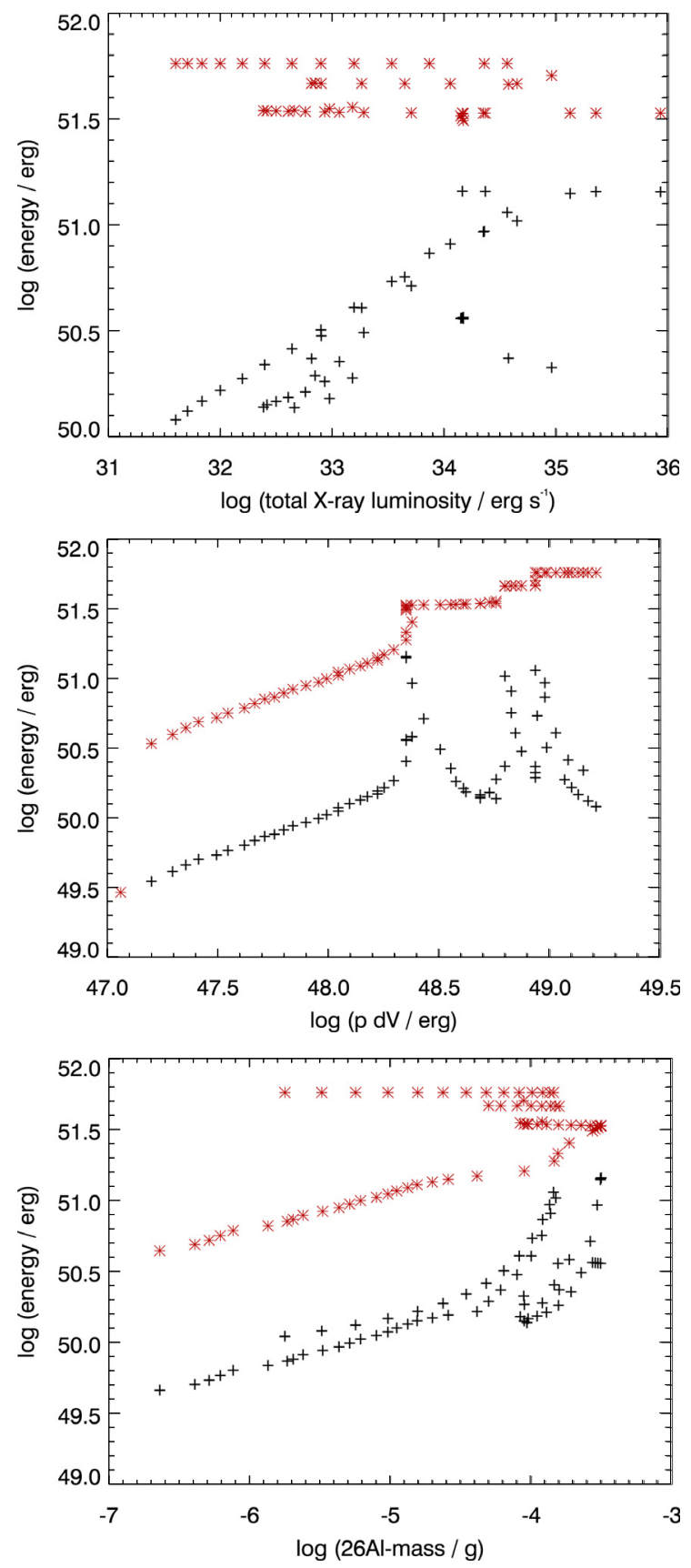

Fig. 11. Scatter plots of the cumulative input energy (red stars) and the total current energy (black pluses), both at a given time, over a potential tracer on the horizontal axis for the representative simulation 3S1-hr. For the latter, we use the X-ray luminosity (top), the work against the ambient pressure (middle), and the mass of the radioactive isotope ${ }^{26} \mathrm{Al}$. See text for details.

the microscopic mixing should be faster than the instabilities we resolve. Nevertheless, mixing of gas phases will introduce a temporary non-equilibrium ionisation structure. This is expected to modify the emissivity of certain emission lines. Effects are however expected mainly in the UV and EUV part of the spectrum, and thus below the soft X-ray regime we consider here (Boehringer \& Hartquist 1987).

We have argued that high X-ray luminosities are a signature of large internal energy content of the superbubbles. It would therefore be interesting to have a comparable observational tracer of the bubble energy. 
In Fig. 11, we compare several possible tracers with the cumulative input energy and the current energy in the simulation box, both at a given time. As expected from our results, the X-ray luminosity does not correlate with the cumulative energy input. For an individual superbubble, it makes therefore no sense to compare the X-ray luminosity to the stellar energy input (in contrast to the case for an ensemble of superbubbles when considering the X-ray luminosity of an entire galaxy, as discussed above). However, the X-ray luminosity does correlate with the current energy, present in a superbubble at any given time, though with some scatter. The work against the ambient pressure may be estimated from HI-holes associated with the superbubbles (e.g. Bagetakos et al. 2011). Here, we defined the superbubble radius from the density peak in the column density map, similar to what one would do for HI observations. The work done by the superbubble correlates very well with the cumulative input energy, but not with the current energy at a given time. Thus, HI-observations may inform us about the total energy released by the relevant group of massive stars and provide us with complementary information with regard to $\mathrm{X}$-rays. Massive stars eject ${ }^{26} \mathrm{Al}$ in their winds and supernovae. ${ }^{26} \mathrm{Al}$ emits a Gamma-ray line which has been used to constrain the stellar content of superbubbles (e.g. Diehl et al. 2010; Voss et al. 2010, 2012), infer the star-formation rate of the Milky Way (Diehl et al. 2006), and as kinematics tracer for hot, recently ejected gas (Kretschmer et al. 2013). We used the data from stellar models as compiled in Voss et al. (2009) to relate the total signal expected from our simulated groups of stars to their mechanical energy output as well as the retained energy at a given time. (Fig. 11, bottom). As expected, the cumulative energy input may vary by more than an order of magnitude for the same ${ }^{26} \mathrm{Al}$-mass. However, as ${ }^{26} \mathrm{Al}$ is correlated to supernovae and strong wind phases, there is a correlation with the current energy of the superbubble, though with some scatter, because the (exponential) radioactive decay differs from the (power law) radiative energy loss of the superbubble. Because of the correlation of the current bubble energy with the X-ray luminosity (compare above), one would essentially expect the ${ }^{26} \mathrm{Al}$-detected superbubbles to be X-ray bright. This is indeed the case for the three individual superbubbles where ${ }^{26} \mathrm{Al}$ has been firmly detected, Cygnus (Cash et al. 1980; Martin et al. 2010; Xu et al. 2013), Orion (Diehl 2002; Voss et al. 2010, and this paper), and Scorpius-Centaurus (Snowden et al. 1997; Diehl et al. 2010).

Our simulations also allow an estimate for the optical emission-line luminosity related to the shocks driven by the superbubble shells. Again, the reason why we can do this estimate is that the energy tracks after each supernova explosion are similar, and the stored energy in a superbubble does not accumulate with the number of explosions (compare also Paper I). In our simulations, 90 per cent of the injected energy is radiated by the shell. Assuming that 7 per cent of this energy is emitted in $\mathrm{H} \alpha$, appropriate for slow shocks (Innes et al. 1987), yields a superbubble-related $\mathrm{H} \alpha$-luminosity of about $L_{\mathrm{SB}}(\mathrm{H} \alpha) \approx 5 \times 10^{40} \mathrm{SFR} \mathrm{erg} \mathrm{s}^{-1}$, where the star-formation rate is taken in units of solar masses per year. This is not the dominant, but still a significant fraction of the observed luminosity $L_{\text {obs }}(\mathrm{H} \alpha)=1.3 \times 10^{41} \mathrm{SFR} \mathrm{erg} \mathrm{s}^{-1}$ (Kennicutt 1998). Thus, while the shock-related part of the $\mathrm{H} \alpha$-luminosity might plausibly trace the current energy content of a superbubble, the signal is likely strongly confused by effects of photoionisation.

Ackermann et al. (2011) use the Fermi satellite to identify a cocoon of cosmic rays in the nearby Cygnus superbubble. Cosmic ray acceleration requires strong shocks (e.g. Vink \& Yamazaki 2014). The strong energy loss in our superbubbles lead to comparatively low temperatures in the bubble interiors and thus increase the probability for newly formed supernova shocks to exceed the critical Mach number. However, since cosmic rays pervade the Galaxy, we do not expect them to loose their energy similarly quickly than the thermal gas in the superbubble. Thus, we would again expect that the cosmic ray signal is more closely related to the total input energy than to the current internal energy of a superbubble.

Therefore, it appears difficult to find another observational tracer with comparable properties to the soft X-ray luminosity, apart from ${ }^{26} \mathrm{Al}$ which is, however, only detectable close by in the Milky Way. Uniquely, soft X-rays trace the current energy content, and not the cumulative mechanical energy.

\section{Conclusions}

From the time-dependence of the energy injection into superbubbles and the 3D nature of the hydrodynamics, we are able to reproduce the basic X-ray properties of superbubbles: strong variation in X-ray luminosities with space and time, peak luminosities of the order of $10^{36} \mathrm{erg} \mathrm{s}^{-1}$, sub-keV temperatures and spatially varying spectral properties, which we relate to global oscillations.

The analogy between the dynamics of superbubbles and the one of winds with constant energy input rate (Weaver et al. 1977), which has been made in the literature, is inadequate in several respects: the X-ray luminosity is strongly linked to the time variability of the energy input rate; consequently, hydrodynamic instabilities and mixing are important. Including these effects, we reproduce the peak luminosities and soft spectra observed in superbubbles.

The X-ray emission from our simulated superbubbles, however, fades too quickly and has slightly too low temperatures. This leads to an underprediction of the diffuse X-ray luminosity of nearby galaxies by about a factor of ten. We suspect that this may be due to suppression of mixing after the shockheating, possibly related to magnetic fields. An alternative explanation would be additional contributions from other sources, e.g. high-mass X-ray binaries (Persic et al. 2004).

We find that the soft X-ray emission probably uniquely traces the current energy content of superbubbles (except for ${ }^{26} \mathrm{Al}$ for nearby objects), whereas other tracers correlate much better with the cumulative mechanical energy input from a group of massive stars.

Acknowledgements. We thank the anonymous referee for useful comments. This research was supported by the cluster of excellence "Origin and Structure of the Universe" (http: //www . universe-cluster.de). We acknowledge the use of NASA's SkyView facility (http://skyview.gsfc.nasa.gov) located at NASA Goddard Space Flight Center.

\section{Appendix A: Effects of numerical resolution}

We repeated run $3 \mathrm{~S} 1 \mathrm{-hr}$ at lower resolution, by reducing the maximum refinement level by one and two, respectively. Figure A.1 shows the X-ray luminosity in various bands over time for the three simulations. Over most of the time, the simulation has converged. Exceptions (for technical reasons) are the $\mathrm{X}$-ray peaks (and minima), because in these short phases, the snapshot closest to the peak is offset in physical time by various amounts. Moderate differences occur in phases of low and 


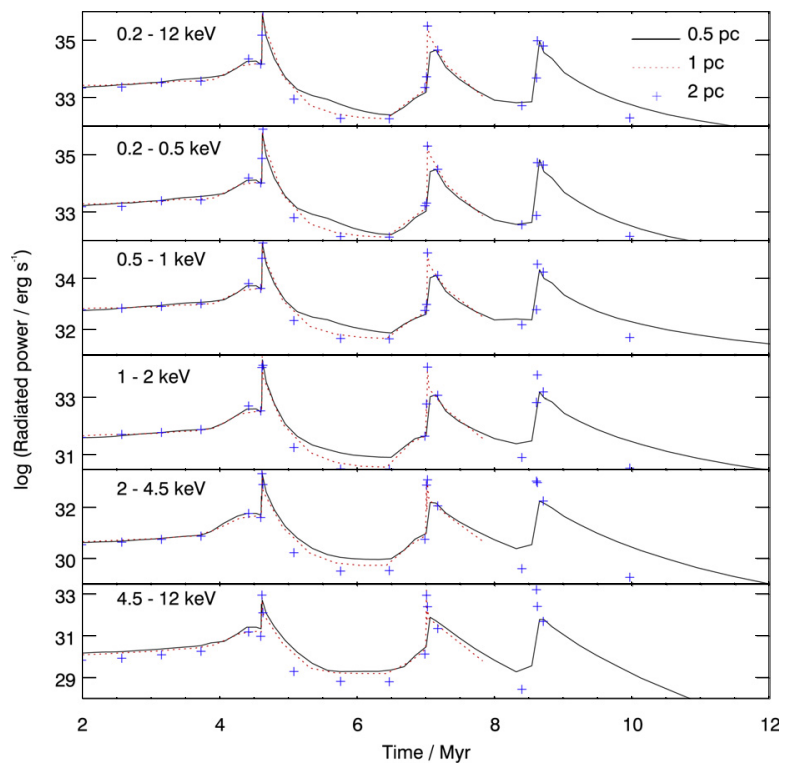

Fig. A.1. Integrated X-ray luminosity over time for different spatial resolutions and different energy bands indicated in the respective individual plots. The solid black line is for the highest resolution run, shown in Fig. 1 ( $0.5 \mathrm{pc}$ resolution for finest AMR level). The dotted red curve is for a twice coarser grid, the blue pluses for again a factor of two worse resolution.

declining luminosities. However, even after these moderate discrepancies the luminosity returns to essentially the same level at each increase in energy input, i.e. whenever the bubble achieves high luminosities.

\section{References}

Ackermann, M., Ajello, M., Allafort, A., et al. 2011, Science, 334, 1103 Ackermann, M., Ajello, M., Allafort, A., et al. 2012, ApJ, 755, 164 Agertz, O., Kravtsov, A. V., Leitner, S. N., \& Gnedin, N. Y. 2013, ApJ, 770, 25 Arnaud, K. A. 1996, in Astronomical Data Analysis Software and Systems V, eds. G. H. Jacoby, \& J. Barnes, ASP Conf. Ser., 101, 17

Bagetakos, I., Brinks, E., Walter, F., et al. 2011, AJ, 141, 23

Bamba, A., Ueno, M., Nakajima, H., \& Koyama, K. 2004, ApJ, 602, 257

Bell, A. R. 2013, Astropart. Phys., 43, 56

Boehringer, H., \& Hartquist, T. W. 1987, MNRAS, 228, 915

Böhringer, H., \& Werner, N. 2010, A\&ARv, 18, 127

Boomsma, R., Oosterloo, T. A., Fraternali, F., van der Hulst, J. M., \& Sancisi, R. 2008, A\&A, 490, 555

Breitschwerdt, D., \& de Avillez, M. A. 2006, A\&A, 452, L1

Brown, A. G. A., Hartmann, D., \& Burton, W. B. 1995, A\&A, 300, 903

Butt, Y. M., \& Bykov, A. M. 2008, ApJ, 677, L21

Bykov, A. M., Gladilin, P. E., \& Osipov, S. M. 2013, MNRAS, 429, 2755

Cash, W., Charles, P., Bowyer, S., et al. 1980, ApJ, 238, L71

Chu, Y.-H. 2008, in IAU Symp. 250, eds. F. Bresolin, P. A. Crowther, \& J. Puls, 341

Creasey, P., Theuns, T., \& Bower, R. G. 2013, MNRAS, 429, 1922

Croton, D. J., Springel, V., White, S. D. M., et al. 2006, MNRAS, 365, 11

Dahlen, T., Strolger, L.-G., Riess, A. G., et al. 2012, ApJ, 757, 70

Dawson, J. R., McClure-Griffiths, N. M., Wong, T., et al. 2013, ApJ, 763, 56

de Avillez, M. A., \& Breitschwerdt, D. 2005, A\&A, 436, 585

Diehl, R. 2002, New Astron. Rev., 46, 547

Diehl, R. 2013, Rep. Prog. Phys., 76, 6301

Diehl, R., Halloin, H., Kretschmer, K., et al. 2006, Nature, 439, 45

Diehl, R., Lang, M. G., Martin, P., et al. 2010, A\&A, 522, A51

Dobbs, C. L., Burkert, A., \& Pringle, J. E. 2011, MNRAS, 417, 1318

Dopita, M. A., \& Sutherland, R. S. 1996, ApJS, 102, 161

Dorman, B., \& Arnaud, K. A. 2001, in Astronomical Data Analysis Software and Systems X, eds. F. R. Harnden, Jr., F. A. Primini, \& H. E. Payne, ASP Conf. Ser., 238, 415

Dunne, B. C., Points, S. D., \& Chu, Y.-H. 2001, ApJS, 136, 119
Ehlerová, S., \& Palouš, J. 2013, A\&A, 550, A23

Ellison, D. C., Slane, P., Patnaude, D. J., \& Bykov, A. M. 2012, ApJ, 744, 39

Elmegreen, B. G., \& Burkert, A. 2010, ApJ, 712, 294

Franceschini, A., Braito, V., Persic, M., et al. 2003, MNRAS, 343, 1181

Freyberg, M. J., \& Egger, R. 1999, in Highlights in X-ray Astronomy Int. Conf., eds. B. Aschenbach, \& M. J. Freyberg, 278

Freyer, T., Hensler, G., \& Yorke, H. W. 2006, ApJ, 638, 262

Gaibler, V., Krause, M., \& Camenzind, M. 2009, MNRAS, 400, 1785

Garn, T., \& Alexander, P. 2009, MNRAS, 394, 105

Gaspari, M., Brighenti, F., \& Temi, P. 2012, MNRAS, 424, 190

Gräfener, G., Vink, J. S., de Koter, A., \& Langer, N. 2011, A\&A, 535, A56

Heald, G. H. 2012, ApJ, 754, L35

Henriques, B. M. B., White, S. D. M., Thomas, P. A., et al. 2013, MNRAS, 431, 3373

Huarte-Espinosa, M., Krause, M., \& Alexander, P. 2011, MNRAS, 417, 382

Innes, D. E., Giddings, J. R., \& Falle, S. A. E. G. 1987, MNRAS, 224, 179

Jaskot, A. E., Strickland, D. K., Oey, M. S., Chu, Y.-H., \& García-Segura, G. 2011, ApJ, 729, 28

Jo, Y.-S., Min, K.-W., Lim, T.-H., \& Seon, K.-I. 2012, ApJ, 756, 38

Jun, B., Norman, M. L., \& Stone, J. M. 1995, ApJ, 453, 332

Kavanagh, P. J., Sasaki, M., \& Points, S. D. 2012, A\&A, 547, A19

Kennicutt, Jr., R. C. 1998, ARA\&A, 36, 189

Knödlseder, J., Cerviño, M., Le Duigou, J.-M., et al. 2002, A\&A, 390, 945

Krause, M. 2005, A\&A, 436, 845

Krause, M. G. H., \& Gaibler, V. 2010, in AGN Feedback in Galaxy Formation, eds. V. Antonuccio-Delogu, \& J. Silk (Cambridge University Press), 183

Krause, M., Fierlinger, K., Diehl, R., et al. 2013, A\&A, 550, A49

Kretschmer, K., Diehl, R., Krause, M., et al. 2013, A\&A, 559, A99

Liedahl, D. A., Osterheld, A. L., \& Goldstein, W. H. 1995, ApJ, 438, L115

Lubos, D. 2012, Master's Thesis, Technische Universität München

Martin, P., Knödlseder, J., Meynet, G., \& Diehl, R. 2010, A\&A, 511, A86

Mewe, R., Gronenschild, E. H. B. M., \& van den Oord, G. H. J. 1985, A\&AS, 62, 197

Mewe, R., Lemen, J. R., \& van den Oord, G. H. J. 1986, A\&AS, 65, 511

Minier, V., Tremblin, P., Hill, T., et al. 2013, A\&A, 550, A50

Motte, F., Zavagno, A., Bontemps, S., et al. 2010, A\&A, 518, L77

Muno, M. P., Law, C., Clark, J. S., et al. 2006, ApJ, 650, 203

Nesvadba, N. P. H., Lehnert, M. D., De Breuck, C., Gilbert, A. M., \& van Breugel, W. 2008, A\&A, 491, 407

Nielsen, K. E., Kober, G. V., Weis, K., et al. 2009, ApJS, 181, 473

Oey, M. S. 2009, in AIP Conf. Ser. 1156, eds. R. K. Smith, S. L. Snowden, \& K. D. Kuntz, 295

Oey, M. S., \& García-Segura, G. 2004, ApJ, 613, 302

Oey, M. S., Clarke, C. J., \& Massey, P. 2001, in Dwarf galaxies and their environment, eds. K. S. de Boer, R.-J. Dettmar, \& U. Klein, 181

Ohlendorf, H., Preibisch, T., Gaczkowski, B., et al. 2012, A\&A, 540, A81

Parizot, E., Marcowith, A., van der Swaluw, E., Bykov, A. M., \& Tatischeff, V. 2004, A\&A, 424, 747

Persic, M., Rephaeli, Y., Braito, V., et al. 2004, A\&A, 419, 849

Piontek, F., \& Steinmetz, M. 2011, MNRAS, 410, 2625

Preibisch, T., Roccatagliata, V., Gaczkowski, B., \& Ratzka, T. 2012, A\&A, 541, A132

Puls, J., Kudritzki, R.-P., Herrero, A., et al. 1996, A\&A, 305, 171

Putman, M. E., Peek, J. E. G., \& Joung, M. R. 2012, ARA\&A, 50, 491

Reynolds, R. J., \& Ogden, P. M. 1979, ApJ, 229, 942

Rieger, F. M., de Oña-Wilhelmi, E., \& Aharonian, F. A. 2013, Frontiers of Physics, 8, 714

Roccatagliata, V., Preibisch, T., Ratzka, T., \& Gaczkowski, B. 2013, A\&A, 554, A6

Romeo Velonà, A. D., Sommer-Larsen, J., Napolitano, N. R., et al. 2013, ApJ, 770,155

Rossa, J., Dettmar, R.-J., Walterbos, R. A. M., \& Norman, C. A. 2004, AJ, 128, 674

Russeil, D., Schneider, N., Anderson, L. D., et al. 2013, A\&A, 554, A42

Russell, S. C., \& Dopita, M. A. 1992, ApJ, 384, 508

Sasaki, M., Haberl, F., \& Pietsch, W. 2002, A\&A, 392, 103

Sasaki, M., Breitschwerdt, D., Baumgartner, V., \& Haberl, F. 2011, A\&A, 528, A136

Scannapieco, C., Tissera, P. B., White, S. D. M., \& Springel, V. 2008, MNRAS, 389,1137

Scannapieco, C., Wadepuhl, M., Parry, O. H., et al. 2012, MNRAS, 423, 1726

Schleicher, D. R. G., \& Beck, R. 2013, A\&A, 556, A142

Schure, K. M., Bell, A. R., O’C Drury, L., \& Bykov, A. M. 2012, Space Sci. Rev., 173,491

Silk, J., \& Mamon, G. A. 2012, Res. Astron. Astrophys., 12, 917

Snowden, S. L., Egger, R., Freyberg, M. J., et al. 1997, ApJ, 485, 125

Sommer-Larsen, J., Götz, M., \& Portinari, L. 2003, ApJ, 596, 47

Stil, J., Wityk, N., Ouyed, R., \& Taylor, A. R. 2009, ApJ, 701, 330 
M. Krause et al.: Emergence of superbubbles - X-ray properties

Strickland, D. K., Heckman, T. M., Colbert, E. J. M., Hoopes, C. G., \& Weaver, K. A. 2004a, ApJS, 151, 193

Strickland, D. K., Heckman, T. M., Colbert, E. J. M., Hoopes, C. G., \& Weaver, K. A. 2004b, ApJ, 606, 829

Sturm, R. 2012, Ph.D. Thesis, Technische Universität München

Sturm, R., Haberl, F., Pietsch, W., et al. 2013, A\&A, 558, A3

Sutherland, R. S., \& Dopita, M. A. 1993, ApJS, 88, 253

Tenorio-Tagle, G., Bodenheimer, P., Franco, J., \& Rozyczka, M. 1990, MNRAS, 244,563

Thornton, K., Gaudlitz, M., Janka, H.-T., \& Steinmetz, M. 1998, ApJ, 500, 95

Toalá, J. A., Guerrero, M. A., Chu, Y.-H., et al. 2012, ApJ, 755, 77

Vink, J. 2012, A\&ARv, 20, 49

Vink, J. S., \& Gräfener, G. 2012, ApJ, 751, L34
Vink, J., \& Yamazaki, R. 2014, ApJ, 780, 125

Voigtländer, P., Kamphuis, P., Marcelin, M., Bomans, D. J., \& Dettmar, R.-J. 2013, A\&A, 554, A133

Voss, R., Diehl, R., Hartmann, D. H., et al. 2009, A\&A, 504, 531

Voss, R., Diehl, R., Vink, J. S., \& Hartmann, D. H. 2010, A\&A, 520, A51

Voss, R., Martin, P., Diehl, R., et al. 2012, A\&A, 539, A66

Weaver, R., McCray, R., Castor, J., Shapiro, P., \& Moore, R. 1977, ApJ, 218, 377

Welsh, B. Y., Sallmen, S., \& Jelinsky, S. 2005, A\&A, 440, 547

Xu, W. F., Gao, X. Y., Han, J. L., \& Liu, F. S. 2013, A\&A, 559, A81

Yamaguchi, H., Sawada, M., \& Bamba, A. 2010, ApJ, 715, 412

Zhekov, S. A., \& Park, S. 2011, ApJ, 728, 135

Zinnecker, H., \& Yorke, H. W. 2007, ARA\&A, 45, 481 